\title{
Solvent extraction of trivalent actinides with di(2-ethylhexyl) phosphoric acid
}

\author{
By R. Takayama ${ }^{1}$, K. Ooe ${ }^{1}$, W. Yahagi ${ }^{1}$, H. Fujisawa ${ }^{1}$, Y. Komori ${ }^{1}$, H. Kikunaga ${ }^{1}$, T. Yoshimura ${ }^{1}$, N. Takahashi ${ }^{1}$, \\ K. Takahisa ${ }^{2}$, H. Haba ${ }^{3}$, Y. Kudou ${ }^{3}$, Y. Ezaki ${ }^{3}$, A. Toyoshima ${ }^{4}$, M. Asai ${ }^{4}$, Y. Nagame ${ }^{4}$, T. Saito ${ }^{5}$, T. Mitsugashira ${ }^{6}$ and \\ A. Shinohara ${ }^{1, *}$ \\ ${ }^{1}$ Graduate School of Science, Osaka University, Toyonaka, Osaka 560-0043, Japan \\ 2 Research Center for Nuclear Physics (RCNP), Osaka University, Ibaraki, Osaka 567-0047, Japan \\ ${ }^{3}$ Nishina Center for Accelerator Based Science, RIKEN, Wako, Saitama 351-0198, Japan \\ 4 Japan Atomic Energy Agency, Tokai, Ibaraki 319-1195, Japan \\ ${ }^{5}$ Radioisotope Research Center, Osaka University, Toyonaka, Osaka 560-0043, Japan \\ ${ }^{6}$ Institute for Materials Research, Tohoku University, Oarai, Ibaraki 311-1313, Japan
}

(Received December 22, 2009; accepted in revised form December 20, 2010)

Actinides / Lanthanides / Solvent extraction / Tetrad effect / HDEHP

\begin{abstract}
Summary. We carried out solvent extraction on the trivalent actinides of $\mathrm{Am}, \mathrm{Cm}, \mathrm{Cf}$, and $\mathrm{Fm}$ and the lanthanides (except Pm) using di(2-ethylhexyl)phosphoric acid (HDEHP) in benzene by a batch method. The extraction constants of the HDEHP complexes for these elements were determined systematically under identical conditions. The tetrad effect was clearly observed in the variation of the extraction constants of the lanthanide series. We found that the extraction constant for Fm(III) is much smaller than that for Dy(III), which have similar ionic radii. The extraction constants for Am(III), Cm(III), and Cf(III) are similar to those for corresponding $\mathrm{Ln}(\mathrm{III})$ having similar ionic radii. Two possibilities that account for the lower extraction constant of Fm(III) is suggested.
\end{abstract}

\section{Introduction}

The lanthanide and actinide series involve the filling of the $4 f$ and $5 f$ subshells, respectively. Unlike the lanthanides, the early members of the actinide series have various oxidation states because $5 f$ electrons are less localized than $4 f$ electrons. Although the stable oxidation state of lanthanides and heavy actinides from Am to $\mathrm{Lr}$ except for No is +3 and their chemical properties depend largely on the ionic radii, there are differences in chemical properties between trivalent lanthanides, Ln(III), and actinides, An(III). For example, soft donor ligands favor bonding to An(III) over $\operatorname{Ln}$ (III) during solvent extraction and ion exchange [1]. Another difference is the effect of $f$ electrons such as the tetrad effect [2], which cannot be explained only by ionic radius effects and is accounted for by the stabilization energy based on the filling of the $f$ orbitals [3]. Therefore, a systematic study of the chemical properties of

*Author for correspondence

(E-mail: shino@chem.sci.osaka-u.ac.jp)
$\mathrm{Am}^{3+}$ to $\mathrm{Lr}^{3+}$ and a comparison of these properties to those of lanthanides is important to determine the effects of $5 f$ electrons.

Plenty of experimental work has been carried out for the extraction of the An(III) and Ln(III) into di(2-ethylhexyl)phosphoric acid (HDEHP) by solvent extraction and column chromatography [4-7]. Peppard et al. reported that the tetrad effect of the An(III) during HDEHP extraction is stronger than that of the $\operatorname{Ln}(\mathrm{III})$ based on a comparison of the separation factors between these series along with their atomic numbers [2]. The separation factors that were compared were measured under various experimental conditions. For a more quantitative consideration, the extraction constants of the same compound containing $\operatorname{Ln}(\mathrm{III})$ or An(III) rather than separation factors should be compared. A systematic study of the extraction constants of the Ln(III) and $\mathrm{An}(\mathrm{III})$, however, has not been carried out. Only a few chemical studies of the heaviest An(III) with atomic numbers $Z \geq 100[6,8]$ have been conducted because of their low production rates in heavy-ion induced nuclear reactions and their short half-lives. No extraction constant values have been determined for the heaviest An(III) into HDEHP yet. It is, therefore, of great importance to investigate the extraction constants of the heavy $\mathrm{An}$ (III) from $\mathrm{Cm}$ (III) to $\mathrm{Lr}$ (III) under identical conditions to evaluate the tetrad effects on the An(III).

In this work, the extraction behavior of Am(III), Cm(III), $\mathrm{Cf}(\mathrm{III})$, and Fm(III) into HDEHP was systematically studied together with the $\operatorname{Ln}(\mathrm{III})$ under identical conditions by a batch method. Because these elements have been reported to be extracted as $\mathrm{M}\left[\mathrm{H}(\mathrm{DEHP})_{2}\right]_{3}(\mathrm{M}=\mathrm{An}$ (III) and $\mathrm{Ln}(\mathrm{III}))$ into organic phases containing HDEHP $[9,10]$, the chemical species of these elements were examined to confirm the extraction reaction and the formation of $\left.\mathrm{M}(\mathrm{HDEHP})_{2}\right)_{3}$ by measuring the variation of extraction probabilities. The extraction constants of the verified extraction reaction for each element were determined by slope analysis. The extraction constant trends for the An(III) were compared with that of the $\operatorname{Ln}(\mathrm{III})$. 


\section{Experimental procedures}

HDEHP was obtained as a 99\% pure material from Nacalai Tesque Inc. All the Ln(III) were supplied by SPEX CertiPrep Inc. as $10 \mathrm{ppm}$ standard solutions. The other chemicals used were commercially available reagents in high purity grades. To prepare the aqueous phase for the extraction of $\operatorname{Ln}(\mathrm{III})$, the standard solutions were evaporated to dryness in PFA beakers and were then separately dissolved using an adequate concentration of $\mathrm{HNO}_{3}$. The organic phase used was $0.08-1.0 \mathrm{M}$ HDEHP in benzene. Six milliliter aliquots of each phase were mixed in a polypropylene tube and the mixture was then shaken in an incubator at $31 \pm 1{ }^{\circ} \mathrm{C}$ for $10 \mathrm{~min}$. After centrifuging the mixture for phase separation, a $5 \mathrm{~mL}$ aliquot of the organic phase was added to a different tube. The organic aliquot was then mixed with $5 \mathrm{~mL}$ of $10 \mathrm{M}$ $\mathrm{HNO}_{3}$ to back-extract $\mathrm{Ln}$ (III) into the $10 \mathrm{M} \mathrm{HNO}_{3}$ solution. After that, $4 \mathrm{~mL}$ of the aqueous phase and the organic phase $\left(10 \mathrm{M} \mathrm{HNO}_{3}\right.$ solution) were evaporated to dryness in PFA beakers and were separately dissolved in $5 \% \mathrm{HNO}_{3}$. The $\mathrm{pH}$ of the equilibrated aqueous phase was measured by titration with 0.1 or $0.025 \mathrm{M} \mathrm{NaOH}$ (Kishida Chemical Co., Ltd.). The distribution ratios were determined by measuring the concentrations of the Ln(III) metals in the aqueous and organic phases by ICP-MS (Agilent 7500s) using a calibration curve method.

For the extraction of the $\mathrm{An}(\mathrm{III}), \mathrm{HNO}_{3}$ solutions containing trace amounts of $\mathrm{Am}, \mathrm{Cm}, \mathrm{Cf}, \mathrm{Ce}$, and Eu were used. ${ }^{139} \mathrm{Ce}$ and ${ }^{152} \mathrm{Eu}$ tracers were also used to confirm the extraction behavior of the $\mathrm{Ln}(\mathrm{III})$ by comparison with the results of the ICP-MS measurements. The extraction procedures were almost the same as those for the extraction of the $\operatorname{Ln}(\mathrm{III})$. The distribution ratios were obtained by measuring the radioactivities of ${ }^{241} \mathrm{Am},{ }^{243} \mathrm{Cm}$ and ${ }^{251} \mathrm{Cf}$ in both phases by $\gamma$-ray spectrometry with high-purity Ge detectors.

For Fm extraction, ${ }^{250} \mathrm{Fm}\left(T_{1 / 2}=30 \mathrm{~min}\right)$ was produced in a ${ }^{238} \mathrm{U}\left({ }^{16} \mathrm{O}, 4 n\right)$ reaction using the AVF cyclotron at the RCNP, Osaka University. ${ }^{151}$ Dy was also produced to compare the extraction behavior of trace amounts of ${ }^{151} \mathrm{Dy}$ with that of non-radioactive Dy. A mixed target of ${ }^{238} \mathrm{U}$ and $\mathrm{Ce}$ was prepared to produce ${ }^{250} \mathrm{Fm}$ and ${ }^{151}$ Dy simultaneously. The reaction products that recoiled out of the target were thermalized in helium gas, attached to $\mathrm{KCl}$ aerosols generated by the sublimation of $\mathrm{KCl}$ powder and then transported using a Teflon capillary to a chemistry laboratory. The transported products were deposited on a Naflon sheet for $30 \mathrm{~min}$ and were then dissolved in $200 \mu \mathrm{L}$ of $0.1 \mathrm{M} \mathrm{HNO}_{3}$. The aqueous phase was mixed with $200 \mu \mathrm{L}$ of a HDEHPbenzene solution. The mixture was shaken in an incubator at $31 \pm 1{ }^{\circ} \mathrm{C}$ for $10 \mathrm{~min}$. After phase separation by centrifugation, $160 \mu \mathrm{L}$ of each phase was separately evaporated to dryness on a Ta dish and was then burned off to completely remove any organic residues from the Ta dish. The Ta dishes were then assayed by $\alpha$-particle spectrometry with a Si PIN diode detector to obtain the distribution ratios.

\section{Results and discussion}

The extraction of trivalent lanthanide and actinide ions with HDEHP is represented by:

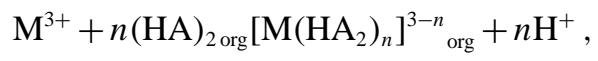

where $\mathrm{M}$ is a lanthanide or actinide and HA and $\mathrm{H}$ represent HDEHP and deprotonated HDEHP (DEHP), respectively $[9,10]$, which forms a dimer in the organic solvent [11]. The distribution radio $D$ of $\mathrm{M}^{3+}$ between the aqueous and organic phases is described as:

$$
D=\left[\mathrm{M}^{3+}\right]_{\mathrm{org}} /\left[\mathrm{M}^{3+}\right]_{\mathrm{aq}} .
$$

The extraction constant $K_{\text {ex }}$ is expressed in terms of the law of mass action and from Eq. (1) is:

$$
K_{\text {ex }}=\left[\mathrm{M}\left(\mathrm{HA}_{2}\right)_{n}\right]_{\text {org }}\left[\mathrm{H}^{+}\right]^{n}{ }_{\text {aq }} /\left[\mathrm{M}^{3+}\right]_{\text {aq }}\left[(\mathrm{HA})_{2}\right]^{n}{ }_{\text {org }} .
$$

Substituting Eq. (3) into Eq. (2), and taking logarithms of both sides results in the following equation:

$$
\log D=\log K_{\text {ex }}-n \log \left[\mathrm{H}^{+}\right]+n \log \left[[\mathrm{HA}]_{2}\right]_{\text {org }} .
$$

Because the metal concentrations were much lower than the initial ligand concentration and the concentration of HDEHP in the aqueous phase was much smaller than that in the organic phase [11], the ligand concentration at equilibrium can be regarded as being equal to the initial concentration.

Fig. 1a shows the variations of the $\log D$ values of the An(III) and Ln(III) as a function of $\mathrm{pH}$ with a $0.5 \mathrm{M}$ concentration of HDEHP in benzene. The $\log D$ values of each element increases with an increase in $\mathrm{pH}$. The solid lines show the results of the fit using Eq. (4) to the data for each element. The theoretical fit gave $n=3$ as the slope for each data set within statistical error indicating that the extracted $\mathrm{M}\left(\mathrm{HA}_{2}\right)_{3}$ species form as shown in Eq. (1) under the given conditions.

In Fig. $1 \mathrm{~b}$, the variation in $\log D$ is shown as a function of (HDEHP $)_{2}$ concentration at a constant $\mathrm{pH}$ of 1 . The log $D$ values increase linearly with a logarithmic increase in the concentration of (HDEHP $)_{2}$; the +3 slope for each element is obtained by fitting Eq. (4) to the $\log D$ vs. $\log \left[(\mathrm{HDEHP})_{2}\right]$ plot, which proves that $n=3$ from Eq. (4). These results show that $n=3$ and unambiguously confirm that the extracted species are $\mathrm{M}\left(\mathrm{HA}_{2}\right)_{3}$ for $\mathrm{Am}(\mathrm{III}), \mathrm{Cm}(\mathrm{III}), \mathrm{Cf}(\mathrm{III})$, and $\mathrm{Fm}(\mathrm{III})$, and each of the studied $\mathrm{Ln}$ (III) under these conditions.

The $K_{\text {ex }}$ values for each $\mathrm{M}\left[\mathrm{HA}_{2}\right]_{3}$ were then reevaluated by fitting Eq. (4) with $n=3$ to the data shown in Fig. 1b. The data shown in Fig. 1a was not used in this fitting procedure because the varied ionic strength for the data are not appropriate for these determinations. The extraction constants of the $\mathrm{Ln}(\mathrm{III})$ and An(III) determined using Eq. (4) are shown in Fig. 2a as a function of atomic number. It is clear that log $K_{\text {ex }}$ for both the $\mathrm{Ln}$ (III) and An(III) increases as the atomic number increases and that the $K_{\text {ex }}$ values of the $\mathrm{Ln}(\mathrm{III})$ are grouped into four sets. These features for the $\mathrm{Ln}$ (III) are in qualitative agreement with previous works $[2,12,13]$.

Peppard et al. reported that the separation factors for the $\mathrm{Ln}$ (III) and $\mathrm{An}$ (III) plotted against atomic number are grouped into four and suggested that the tetrad effect for the $\mathrm{An}(\mathrm{III})$ is stronger than that for the $\mathrm{Ln}(\mathrm{III})$ [2]. It is, however, reasonable to plot $K_{\text {ex }}$ values against an inverse square of the ionic radius to take into account the surface charge density of the cations to determine the strength of 

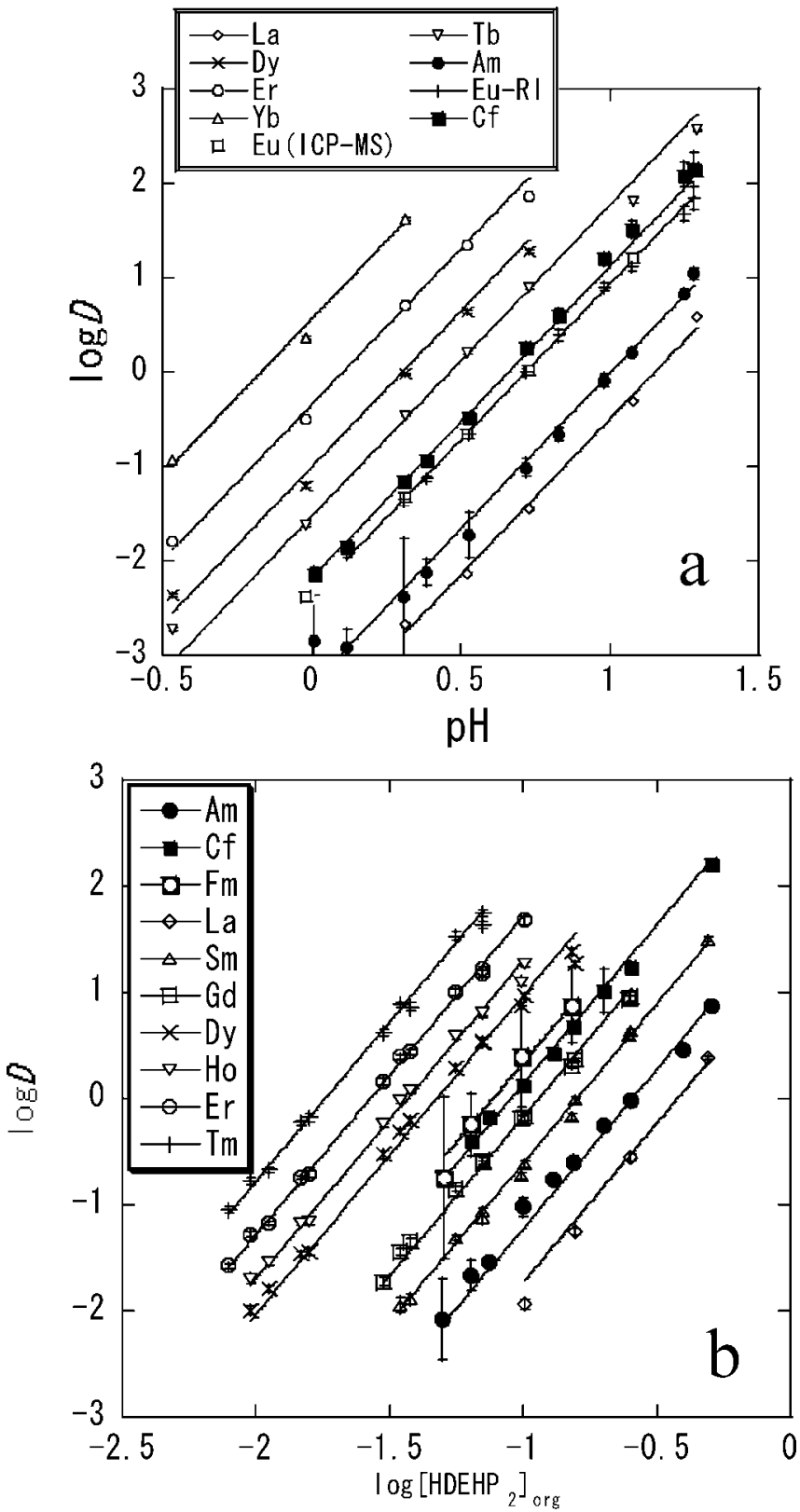

Fig. 1. Variations of $\log D$ against (a) $\mathrm{pH}$ and (b) $\log \left[(\mathrm{HDEHP})_{2}\right]$. Experimental conditions for the aqueous phase: (a) $\mathrm{HNO}_{3}$ and (b) $0.1 \mathrm{M}$ $\mathrm{HNO}_{3}$, and for organic phase: (a) $0.5 \mathrm{M} \mathrm{HDEHP}$ in benzene and (b) HDEHP in benzene.

the tetrad effect from the two data series. The extraction probabilities and/or complex formation of the $\mathrm{Ln}$ (III) and An(III) generally reveal a strong dependence on their surface charges because of the electrostatic interaction of metallic ions to their chemical surroundings. Fig. $2 b$ shows the variation of $K_{\mathrm{ex}}$ as a function of ionic radius. Although no experimentally determined coordination numbers $(\mathrm{CNs})$ exist for $\mathrm{Fm}(\mathrm{III})$, its ionic radius with a $\mathrm{CN}=6$ [14] was adopted. Brïchle et al. [14] determined the ionic radius of Fm(III) from its elution position in cation-exchange chromatography with $\alpha$-hydroxyisobutyric ( $\alpha$-HIB) acid using the linear relationship between the elution positions in $\alpha$ HIB and the ionic radii observed for the $\operatorname{Ln}(\mathrm{III})$. They also compared the decreasing ionic radius trend from $\mathrm{Cf}(\mathrm{III})$ to $\mathrm{Md}(\mathrm{III})$ in addition to those of the heavy $\mathrm{Ln}$ (III) using theoretical calculations and showed that the experimental results
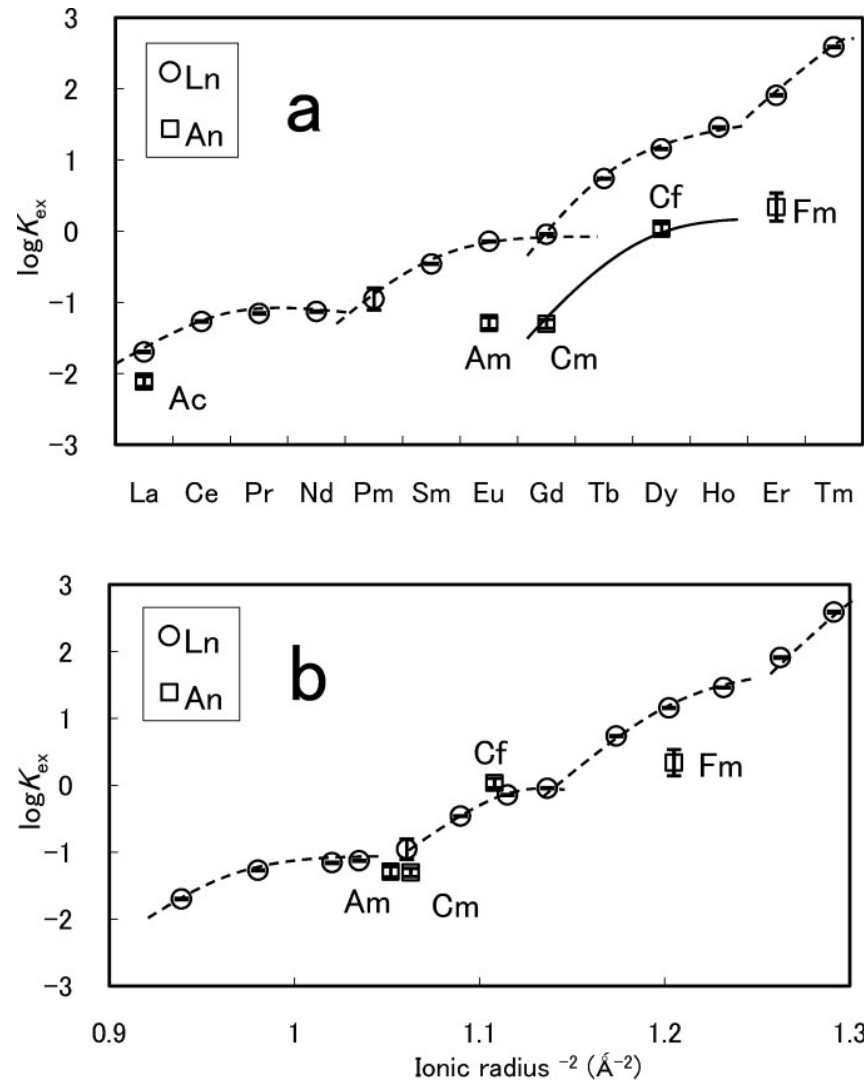

Fig. 2. Variation of $\log K_{\mathrm{ex}}$ as a function of (a) atomic number and (b) ionic radius $[14,15]$.

were consistent with the calculations. The determined ionic radius of Fm(III) is, therefore, the most accurate value at present. In Fig. 2b, clear trends for increasing $\log K_{\text {ex }}$ values are apparent for the $\mathrm{Ln}(\mathrm{III})$ and $\mathrm{An}(\mathrm{III})$ series. $\mathrm{Cf}(\mathrm{III})$ and $\mathrm{Eu}(\mathrm{III})$ have similar ionic radii but the $K_{\text {ex }}$ value of $\mathrm{Cf}$ (III) is slightly larger than that of $\mathrm{Eu}(\mathrm{III})$. On the other hand, $\mathrm{Am}, \mathrm{Cm}$, and Fm are less extracted than their corresponding lanthanides with similar ionic radii. In particular, the $K_{\text {ex }}$ value for Fm(III) is much smaller than the corresponding lanthanide Dy(III). Two possibilities account for the lower extraction constant of Fm(III). One is stronger stabilization, i.e., lowering of the $K_{\mathrm{ex}}$ value for Fm(III) at the third node in the tetrad of An(III) because of the stronger tetrad effect for the heavy An(III) than that for the heavy Ln(III). However, the ionic radius determined for Fm(III) [14] might not be accurate. The other possibility is a greater lowering of the $K_{\mathrm{ex}}$ values for the heavy An(III) from Es(III) to $\operatorname{Lr}(\mathrm{III})$ compared with that of the $\operatorname{Ln}(\mathrm{III})$ where the dependence of the $\mathrm{An}(\mathrm{III})$ extraction probabilities on their ionic radii would be smaller than that of the $\mathrm{Ln}(\mathrm{III})$. In future, we will determine the extraction constants of Bk(III), Es(III), Md(III) and $\operatorname{Lr}(\mathrm{III})$ because they are of great importance in determining the deviated extraction constant of Fm(III).

\section{Conclusion}

The extraction constants for Am(III), Cm(III), Cf(III), Fm(III), and $\mathrm{Ln}$ (III) with HDEHP were determined. Increasing trends were clearly observed for the extraction constants 
of the $\mathrm{An}(\mathrm{III})$ and the $\mathrm{Ln}(\mathrm{III})$ with a decrease in the ionic radius. The $K_{\mathrm{ex}}$ value of Fm(III) was much smaller than that of Dy, which had a similar ionic radius. This implies that the heavy An(III) have a stronger tetrad effect than the Ln(III) or that the $A n($ III) and $\operatorname{Ln}($ III) have a different dependence of extraction constants on ionic radii.

Acknowledgment. This study was carried out under the Cooperative Research Program of the International Research Center for Nuclear Materials Science, Institute for Materials Research (IMR), Tohoku University. The authors would like to thank the technical staff of the Research Center for Nuclear Physics, Osaka University for their excellent operation of the accelerator.

\section{References}

1. Jensen, M. P., Bond, A. H.: Comparison of covalency in the complexes of trivalent actinide and lanthanide cations. J. Am. Chem. Soc. 124, 9870 (2002).

2. Peppard, D. F., Bloomquist, C. A. A., Horwitz, E. P., Lewey, S., Mason, G. W.: Analogous actinide (III) and lanthanide (III) tetrad effects. J. Inorg. Nucl. Chem. 32, 339 (1970).

3. Kawabe, I.: Lanthanide tetrad effect in the $\mathrm{Ln}^{3+}$ ionin radii and refined spin-pairing energy theory. Geochem. J. 26, 309 (1992).

4. Peppard, D. F., Mason, G. W., Maier, J. L., Driscoll, W. J.: Fractional extraction of the lanthanides as their di-alkyl orthophosphates. J. Inorg. Nucl. Chem. 4, 334 (1957).

5. Peppard, D. F., Mason, G. W., Driscoll, W. J.,Sironen, R. J.: Acidic esters of orthophosphoric acid as selective extractants for metallic cations-tracer studies. J. Inorg. Nucl. Chem. 7, 276 (1958).
6. Horwitz, E. P., Bloomquist, C. A. A.: Chemical separation for super-heavy element searches in irradiated uranium targets. J. Inorg. Nucl. Chem. 37, 425 (1975).

7. Sato, T., Ueda, M.: The extraction of yttrium(III) and lanthanum(III) from hydrochloric acid solution by di-(2-ethylhexyl) phosphoric acid. J. Inorg. Nucl. Chem. 35, 1003 (1973).

8. Gavrilov, K. A., Gvuzdz, E., Stary, J., Wang Tung Seng: Investigation of the solvent extraction of californium, fermium and mendelevium. Talanta 13, 471 (1966).

9. Kosyakov, V. N., Yerin, E. A.: Separation of transplutonium and rare-earth elements by extraction with HDEHP from DTPA solutions. J. Radioanal. Chem. 43, 37 (1978).

10. Aguilar, M., Liem, D. H.: Studies on the solvent extraction of europium(III) by di-(2-ethylhexyl) phosphoric acid (HDEHP) in toluene. Acta. Chem. Scand. A30, 313 (1976).

11. Liem, D. H.: Studies of the complex formation between di-2ethylhexyl-phosphate (HDEHP) and tributylphosphate (TBP) or trioctylamine (TOA) in toluene. Acta. Chem. Scand. 26, 191 (1972).

12. Janowski, A., Lewandowski, W.: Investigations of the doubledouble effect by IR spectroscopy. J. Inorg. Nucl. Chem. 42, 561 (1980).

13. Harmon, H. D., Peterson, J. R.: The tetrad effect: the thiocyanate complex stability constants of some trivarent actinides. J. Inorg. Nucl. Chem. 34, 1381 (1972).

14. Brüchle, W., Schädel, M., Scherer, U. W., Kratz, J. V., Gregorich, K. E., Lee, D., Nurmia, M., Chasteler, R. M., Hall, H. L., Henderson, R. A., Hoffman, D. C.: The hydration enthalpies of $\mathrm{Md}^{3+}$ and $\mathrm{Lr}^{3+}$. Inorg. Chim. Acta. 146, 267 (1988).

15. Shannon, R. D.: Revised effective ionin radii and systematic studies of interatomic distances in halides and chalcogenides. Acta Cryst. A32, 751 (1976). 